\title{
Ulrich Saxer Gestaltung von Realität durch Public Relations
}

„Menschen konstruieren ihre Wirklichkeit subjektiv und eigenverantwortlich. Es gibt demnach so viele Wirklichkeiten, wie es Menschen gibt" (Merten 2005b, S. 136). Hielten sich PR-Spezialisten und Journalisten an dieses „Basistheorem des Konstruktivismus“, wäre für beider und der Gesellschaft Zukunft Schlimmes zu gewärtigen. Glücklicherweise ist es den wenigsten unter ihnen geläufig, und so konstruieren sie weiterhin mehr oder weniger erfolgsgewiss Wirklichkeiten für andere gemäß den Regeln ihrer Öffentlichkeitsberufe, Medien- und PR-Realitäten, auf dass diese ihre Wirklichkeit auch diejenige ihrer Rezipienten werde.

Was ja bei jedem gewichtigeren politischen, wirtschaftlichen oder kulturellen Anlass, Parteitag, Firmenjubiläum, Festival oder Fußballturnier von ihnen praktiziert wird, ist "Wirklichkeitsmanagement" (Merten 2005a, S. 21ff.) in immer größerem Stil. Dadurch wird eine allgemeine gesellschaftshistorische Entwicklung intensiviert, die auf die folgende These gebracht werden kann:

\section{Realitätsstabilisierung als Erfahrung}

Die Verunsicherung des Wirklichkeitsbewusstseins stellt neben der funktionalen Differenzierung, dem institutionellen Wandel und der Individualisierung einen weiteren Metatrend dar, der moderne Gesellschaften und ihre Mitglieder elementar prägt. Dieser gründet vor allem im Nebeneinander verschiedenster Realitätsdefinitionen von beschränkter allgemeiner Verbindlichkeit, die zunehmend auch als Konstruktionen erkannt werden. Entsprechend weit ist der Kreis der Wettbewerber, darunter vor allem die Massenmedien und PR als Kommunikationssystem, um Akzeptanz ihrer Realitätsdefinitionen.

"Gibt es eigentlich die Wirklichkeit noch?“ (Meckel 2002, S.31ff.) wurde denn auch schon 2002 von kommunikationswissenschaftlicher Seite gefragt, und eine echt konstruktivistische Antwort darauf lautet: „Erzeugung von Fakten durch Relativierung von Fiktionen“" (Merten 2002, S.36ff.). Welches ist nur schon die Wirklichkeit des Fußballs, die „wirkliche“: das allüberall und auch anlässlich der europäischen Fussballmeisterschaft in Österreich und in der Schweiz 
propagierte Fairplay, das Milliardengeschäft, die nationale Selbstbestätigung (dank so und so vielen ausländischen Kickern im vaterländischen Team), die europäische Völkerverständigung im Zeichen des runden Leders oder die neue Völkerschlacht in Wien, Basel und weiteren Stadien? "If people define situations as real, they are real in their consequences" sagt das so genannte Thomastheorem, die fundamentalste sozialpsychologische Erkenntnis, und der darin festgehaltene Mechanismus hat zur Folge, dass eben auch Fiktionen zu Realitäten werden können, wie 1938 der als Nachrichtensendung präsentierte fingierte Überfall von Marsmenschen auf die Erde in Orson Welles' berüchtigtem Radioschocker in den Köpfen unzähliger Hörer(innen), die dadurch in Panik gerieten und flüchtend die Straßen verstopften.

Für den Großteil der Kommunikationswissenschaftler kommt mittlerweile Wirklichkeit als dasjenige, was „wirklich" geschieht und was von Medien beobachtet wird bzw. worüber sie berichten, ins Spiel. Realität in diesem umfassenden Sinn ist all das, was je vorhanden war, vorhanden ist, vorhanden sein wird. Realität in diesem Verständnis enthält potenziell unendlich viele verschiedene Informationen" (Bentele 2006, S. 312) und kann demzufolge immer nur selektiv erfasst und kommuniziert werden.

Die Mitglieder der funktional differenzierten hochkomplexen modernen Gesellschaften erfahren Realität in immer kleineren Ausschnitten direkt oder eben als die Pseudototalität der TV-Tagesschauen und immer weniger als durch Tradition, namentlich kirchliche, oder sonst institutionell verbindlich geordnete, stattdessen als verwirrend wandelbar, und wie die Glaubwürdigkeitseinbußen der Massenmedien belegen, schrumpft allenthalben das Vertrauen in die Verlässlichkeit von Medienrealität. Trotzdem formulieren Massenmedien auch noch in der Ära von Multimedia die am allermeisten diffundierten und rezipierten Realitätsdefinitionen von eben mehr oder minder akzeptierter Verbindlichkeit und bilden daher nach wie vor die Hauptzielobjekte von PR-Strategien. Entscheidend ist, dass Massenmedien Realität als Ereigniswelt und Themenuniversum gemäß Nachrichtenwerten präsentieren, und unter den letzteren figurieren prominent Aggression, Kontroverse, Ungewissheit und Überraschung, aber auch Konsonanzwerte wie Stereotypie, Vorhersehbarkeit und Themenbündelung.

Diese massenmediale Ereigniswelt konstituiert also eine von praktisch jedermann in modernen Gesellschaften ständig genutzte Offerte, Realität als zugleich überraschende, beunruhigende und doch wieder kalkulierbare, sie und ihn bestätigende, aber jedenfalls fragmentierte Szenenfolge wahrzunehmen und geistig zu rekonstruieren. 
Eine gängige Typologie unterscheidet dabei natürliche, soziale und Medienereignisse, also Frühlingserwachen und Erdbeben, durch menschliches Handeln bewirktes Geschehen und Inszenierungen zu Ehren der Massenmedien. Und mit dieser dreigegliederten Ereigniswelt, dieser kaleidoskopischen Abfolge von Akteuren und Themen und von verschiedenen Definitionen dessen, was wichtig und gut sei mit all dem dauernd konfrontiert sind Gesellschaftsmitglieder mit geschwächten nachbarlichen und verwandtschaftlichen Primärbindungen, durch intensivierte Arbeitsteilung zur Flexibilität verurteilt und im Gefolge neuzeitlicher Individualisierung auf sich selbst gestellte Interpreten der oder von Wirklichkeit. Und hier hakt die zentrale, aber von der PR-Wissenschaft zu wenig gewürdigte Leistung von Public Relations ein, die thesenhaft folgendermassen umschrieben werden kann:

\section{Realitätsvergewisserung durch PR}

Dass PR mittlerweile praktisch sämtliche Gesellschaftsbereiche kommunikativ erschließen helfen, hängt maßgeblich mit dieser Realitätslabilisierung zusammen. Sie führt als zentrales Problem zur Ausdifferenzierung entsprechender Lösungsstrukturen, darunter dieses Strategiesystem kommunikativer Wirklichkeitsvergewisserung als Orientierungs-, Handlungs- und mentales Stabilisierungsinstrument. Professionelle PR heben ja wichtige Gesellschaftsbelange ins öffentliche Bewusstsein, lassen relevante Interessen zu Wort kommen und schaffen über die Konstituierung entsprechender Öffentlichkeiten Grundlagen und Vertrauen für gemeinsames Handeln. Dazu entwickeln sie eine wachsende Vielfalt von Kommunikationstechniken, um unterschiedliche Orientierungshorizonte miteinander zu verbinden.

Das Wissen, ja das Vertrauen, in einer konsentierten Wirklichkeit zu leben und zu agieren, ist für zielgerichtetes individuelles und kollektives Handeln unerlässlich, wenn auch eben im Widerspruch zum täglich erfahrenen Pluralismus moderner Wirklichkeitskonzeptionen, von einer durch Mobilität und Globalisierung entgrenzten Welt, einer des alles technisch Machbaren oder der ökologisch bedrohten Erde etc. Die PR-Aufgabe der Wirklichkeitsvergewisserung entbehrt jedenfalls nicht der Paradoxien.

Sie impliziert einmal, überhaupt ein Bewusstsein für die Mannigfaltigkeit des gesellschaftlichen Geschehens zu schaffen und namentlich auch unterbelichtete, aber relevante Aspekte der Realität öffentlich zu machen. Dies leisten namentlich die Non-Profit-PR oder auch kommunale Öffentlichkeitsarbeit. Da mit der Zunahme der Individualisierung auch die Privatisierung von Mentalitäten und Ein- 
stellungen einhergeht, auf die wiederum mehr und mehr Angebote privater Rundfunksender, spezialisierte Zeitschriften und Gratistitel ausgerichtet sind, haben unspektakuläre Gesellschaftsanliegen oder wenig einflussreiche Minoritäten vermehrt Mühe, in der Medienrealität überhaupt aufzuscheinen und damit gegebenenfalls breitenwirksam Handlungsbedarf zu signalisieren. In dieser Hinsicht erwachsen mithin der Öffentlichkeitsarbeit wichtige komplementäre Aufgaben.

Effiziente Artikulation von Interessen, zumal in Konkurrenz mit immer mehr anderen und in hochfragmentisierten Öffentlichkeiten, setzt in wachsendem Maß organisatorische und kommunikative Kompetenz und entsprechende Mittel voraus, solche selbst zu entwickeln oder einzukaufen, eben bei PR-Spezialisten. Dies kann freilich implizieren, dass gesellschaftliche Realitätsvergewisserung durch PR, also die von Öffentlichkeitsarbeit mit besonderem Verbindlichkeitsanspruch konstituierte, eine plutokratische wird, vom Gott des Reichtums Pluto gemäß seinen Präferenzen diktiert. Glücklicherweise ist aber kreative Phantasie, Interessen öffentlich wirksam zu präsentieren, nicht unbedingt an große PR-Etats gebunden - wie schon vor 40 Jahren von findigen Theaterdramaturgen studentischen Protestes demonstriert.

Ihr Endziel der Beziehungsoptimierung vermögen PR aber bekanntlich nur über vielstufige Strategien zu erreichen. Beziehungsoptimierung durch Kommunikation indes setzt die Etablierung einer vertrauenswürdigen gemeinsamen Realitätsbasis zwischen den Auftraggebern von Gesundheitskampagnen, Unternehmen etc. bzw. ihren PR-Spezialisten und den jeweiligen Zielöffentlichkeiten voraus. Und hier kommt eben die Problematik der unterschiedlichen Wirklichkeitskonzeptionen, der je anderen Bezugsrahmen (frames), unter denen die letzteren die Welt und etwaige PR-Anliegen erleben, voll zum Tragen. Nicht allein die Verknappung öffentlicher Aufmerksamkeit unter dem Ansturm von immer mehr Bewerbern um diese gilt es $z u$ bedenken, sondern dass Mitglieder moderner Gesellschaften mehr denn je der Vergewisserung bedürfen, was ihnen da von PR-Seite unterbreitet wird, habe ein vertrauenswürdiges, einsehund nachvollziehbares Fundament in der Wirklichkeit - der ihren! Nur dann wird Realität durch PR in weiteren Schritten zielgerecht gestaltbar.

Darum kann die Inszenierung von Ereignissen, die von den Medien zu Medienereignissen geadelt werden, nur als eine Hauptstrategie praktiziert werden, aber nicht als das Endziel von PR gelten, auch wenn dies bei Organisationen wie Greenpeace der Fall sein mag. 
Dem Event-Management eignet wie jeder Strategie ein spezifisches, aber kein allseitiges Problemlösungsvermögen. Dieses lässt sich zur folgenden These verdichten:

\section{Event-Management als Hauptstrategie}

Durch die Inszenierung von Realität als Ereignis bzw. Event suchen immer mehr Akteure öffentliche Aufmerksamkeit und Zustimmung für ihre Anliegen zu wecken. Event-Marketing mit Hilfe von PR-Professionals wird als Strategie, öffentliche Bedeutsamkeit, ja Dringlichkeit zu signalisieren, in modernen Gesellschaften über das System Wirtschaft hinaus auch generell in der Politik, als symbolische Politik, und der Kultur, als Happening, praktiziert. Die Grundintention dieser Events, nämlich Medienpublizität für bestimmte Interessen zu erwirken, wird allerdings von den PR-Professionals möglichst verheimlicht, die Transparenznorm medialer Berichterstattung damit durchkreuzt. Zudem ist die Gefahr erheblich, dass das Event die Botschaft zu sehr überlagert, und überdies ist Impression Management, Imagegenerierung durch Event-Marketing risikoreich.

Für die Berechtigung, ja Notwendigkeit, moderne Gesellschaften als Mediengesellschaften zu klassifizieren, zeugt maßgeblich auch die rasante gesamtgesellschaftliche Vermehrung von Medienereignissen: von den pressegesponserten Segelregatten im 19. Jahrhundert über die selbstverständlich gewordenen Medienkonferenzen für alles und jedes bis zur gezielten Maximierung von Nachrichtenfaktoren bei der Organisation sportlicher Großanlässe wie Olympischen Spielen oder Politikerwahlen. Was sich hier vollzieht, ist letztlich nicht weniger als die Verkehrung der Ereigniskausalität, als die ursprünglichen Vermittler, eben die Medien, in Medienereignissen zu Hauptakteuren werden, und die es früher waren, die Staatsmänner, Wirtschaftskapitäne und Künstlergrößen amtieren sozusagen als beflissene Ausführungsgehilfen der Anweisungen der Medienregisseure.

Moralisierend hier von „Pseudo-Ereignissen“ zu sprechen, macht allerdings wenig Sinn, denn sobald Interaktionen öffentlich stattfinden, werden sie bis zu einem gewissen Grade auch inszeniert, vollziehen sich nicht wie Tsunamis gemäß Naturgesetzen. Die Entwicklungen, die sich hierin auftun, wollen aber schon ernsthaft bedacht sein: Die von Klaus Merten beschworene Verwandlung von Fakten in Fiktionen zeichnet sich ab, ja der Untergang der vom Journalismus geheiligten Faktizität, die Virtualisierung der Welt, die schon vor vielen Jahrzehnten der Kulturphilosoph Günther Anders voraussah: „Die Welt als Phantom und Matrize“ (Anders 1956). 
Im System Politik und zumal in der politischen Kommunikation sind das Ausmaß und die Tragweite der Eventisierung der Prozesse besonders groß. Das Schwergewicht politischer Kommunikation verlagert sich ja dabei von der Vermittlung politischer Bedeutung auf die Generierung und Präsentation von spektakulären Ereignissen, von der Herbeiführung und Implementation allgemeinverbindlicher Entscheidungen, der Hauptfunktion von Politik, auf deren symbolische Repräsentation, vielfach als Ersatz von politischen Problemlösungen. Enttäuschung am politischen Betrieb und politische Entfremdung sind dabei programmiert, wenn Politiker symbolisch unbegrenzte Handlungsfähigkeit zur Schau stellen, die ihnen im stark vorgespurten politischen Alltag gar nicht zusteht.

Die Eventisierung von Politik dient nämlich in wachsendem Maß auch ihrer Umwandlung in Unterhaltung, in Politainment. Eventpolitik ist nicht nur in den USA, sondern auch in Europa eine Metapolitik des Zeigens vor dem Realisieren, nämlich von Sachpolitik, geworden. Allerdings haben sich die Politik, der Staat seit eh und je in öffentlichen Feiern symbolisch vergegenwärtigt, an nationalen Festtagen Identität zelebriert und die eigenen Leistungen glorifiziert. Und mittlerweile ist bei schrumpfender Verbindlichkeit der politischen Verfasstheit der individualisierten Staatsbürgerschaft die mediendemokratische Eventpolitik außer in den politisch-institutionellen immer stärker auch in den unterhaltungskulturellen Kontext eingebunden worden. An den amerikanischen politischen Veranstaltungen sind bekanntlich die Beinchen schwingenden Majorettes längst die schönste Attraktion.

Selbst in der Kultur, in jenem Funktionssystem, das den gesellschaftlich dominanten Sinn, die Vorstellungen von gut und böse, wichtig und unwichtig, schön und hässlich generiert, also die geistige Strukturierung der Gesellschaftsmitglieder besorgt, ist Event-Marketing, wenn auch vielleicht unter vornehmeren Namen, mehr und mehr gängige Praxis geworden, Aufmerksamkeit, ja Begeisterung und hoffentlich Nachfrage zu wecken. Wenn das investierte Kapital wegen der bekannten „Kostenkrankheit" der Produktion von anspruchsvoller Kultur im Gefolge des damit verbundenen hohen wirtschaftlichen und geistigen Rezeptionsaufwandes schon zu wenig profitabel arbeitet, soll es wenigstens Aufmerksamkeits- und Reputationsdividenden abwerfen, als Happening zum Mediahype auswachsen.

Auch die katholische Kirche, schon von ihrer Tradition her zur ereignishaften Verlebendigung und Symbolisierung ihres Kults bestens qualifiziert, setzt in der Moderne voll auf die neuen Medien der Massenkommunikation und die missionierende Kraft von Medien- 
ereignissen. Allen voran ist hier der unermüdlich und weltweit pilgernd missionierende charismatische Papst Johannes Paul II. als Meister des religiösen Event-Marketings zu nennen, jeweils nach Verlassen des Flugzeugs zur Begrüßung fernsehwirksam den Boden, die Erde jedes neuen Territoriums küssend. Und um ihn und hinter ihm seine publizistisch versierte Entourage aus dem Episkopat in mehr oder minder heftigem Clinch mit den Journalisten, welche ausgestrahlte Fernsehrealität des Papstbesuches die „richtige“ sei (Dorsch-Jungsberger/Roegele/Stolte 1985).

Es ist ja auch die mediale Bereitstellungsqualität als solche, zumal das zunehmend totale Vergegenwärtigungsvermögen der audiovisuellen Medien bis hin zu den Berührungserlebnissen im Cyberspace, das medienexterne Realität fortlaufend in mehr oder minder packende Szenen verwandelt, so attraktive jedenfalls, dass die Großen und Kleinen eifrig mitspielen: die Großen wohlwollend oder als Politiker chronisch staatsmännisch und/oder humorvoll, und die Kleinen, das Fußvolk, meinungsbereit $z u$ allem und jedem, erwiesenermaßen auch zu politischen Vorlagen, die es gar nicht gibt, die aber als journalistischer Jux Rezipienten zu Albernheiten vor dem Mikrofon verleitet. Die Fernsehkamera im Museum, andererseits, dynamisiert hektisch die stillen, regungslosen Gemälde an den Wänden, und Theodor W. Adorno hat einmal treffend bemerkt, die Bücher sähen bereits so aus, als schämten sie sich, keine Filme zu sein. So präsentiert sich die Eventisierung der Realität gewissermaßen als unser aller Gesamtkunstwerk, denn auch wir als Publikum machen mit, weil wir in unserer Individualisiertheit wo immer Partizipation suchen und in unserer Anonymität Andy Warhols sprichwörtliche Viertelstunde "Jedermannsberühmtheit" vor der Kamera als Talkshower oder Meinungskundgeber einziehen.

Trotz allem ist Event-Marketing in der modernen Gesellschaft in mancher Hinsicht funktional, personen- und systemdienlich. Sonst würde auch nicht allenthalben so viel in diese Strategie investiert und würde sie auch nicht in so breitem Maß akzeptiert. Sie vermag eben zumindest temporär Gemeinschaft durch Zuwendung zum Gleichen $\mathrm{zu}$ stiften, das sonst von Externen weitgehend unbeachtet bliebe, z. B. Kirchen- oder Parteitage. Zu Recht legendär ist da etwa der Parteitag der deutschen Sozialdemokraten von 1998, der die Kür des Kanzlerkandidaten so perfekt eventisierte, dass er als Leipziger „Krönungsmesse" in die Fachliteratur (Brosda 1999) eingegangen ist.

Im übrigen gibt es schon ganze Manuale, wie hohe Medienresonanz durch Event-Marketing wahrscheinlich gemacht werden kann. Dabei sichern eben ins Event eingebaute Nachrichtenwerte wie Kon- 
fliktinszenierung oder Personalisierung ebenso Medienbeachtung wie Gewalt oder auch symbolträchtige Örtlichkeiten. Dennoch ist die Strategie risikoreich, und zwar aus verschiedenen Gründen: Erstens, die emotionalen und kognitiven Effekte, die PR-Events hervorrufen, sind nur sehr beschränkt kalkulierbar, auch wenn intensiv vorgetestet wurde. Die jeweilige Ereignis- und Rezeptionssituation ist ja immer wieder eine andere und als ganze anders als im Labor unkontrollierbar komplex, dass zielgerechtes Impression Management durch Ereignismanagement weitgehend Glücksache ist. Zweitens gibt es wohl Erfahrungswerte, namentlich eben die Nachrichtenfaktoren, welche Ereignisse am ehesten Medienresonanz erzeugen, aber damit ist die Rezeption durch das Zielpublikum keineswegs garantiert, und auch deren Qualität nicht. Drittens, da ein deutlich direktiver Gestus von Kampagnenevents Zielöffentlichkeiten eher abschreckt, muss die persuasive Intention des Event-Marketings verschleiert werden, was zu missverständlichen Interpretationen des Events durch die Rezipienten führen kann.

Realitätsgestaltung durch Event-Marketing ist denn auch auf jeden Fall ergänzungsbedürftig, weshalb eine Vielzahl von eigenständigen oder die Hauptstrategie flankierenden Techniken von Realitätsgestaltung durch PR entwickelt worden ist. Drei besonders wichtige werden in der nachfolgenden These angesprochen, und zwar unter dem Gesamtziel der Etablierung "wünschenswerter Wirklichkeiten“ (Merten/Westerbarkey 1994, S. 208):

\section{Wünschenswerte Wirklichkeiten durch PR}

$P R$ suchen wünschenswerte Wirklichkeiten zu etablieren, indem sie das Image ihres Objektes optimieren, dasjenige von Konkurrenten schwächen und Situationen in diesem Sinne kommunikativ kontrollieren. Impression Management, Negative Campaigning und Issues Management sind die entsprechenden Strategien, mit denen diese Ziele anvisiert werden. Alle drei haben dabei besondere Probleme zu meistern: Das erstere, weil Images, Geltungsbilder komplexe und labile sozialpsychologische Gebilde sind; Negative Campaigning, weil dessen Erfolg kulturabhängig ist; und die Optimierung von Risiko- und Konfliktkommunikation bereitet darum Schwierigkeiten, weil diese zentralen Issues sehr vielfältig begründet sind.

Auch das Problemlösungsvermögen dieser Kommunikationsstrategien ist natürlich beschränkt, obwohl PR-Praktiker, die sich ihrer bedienen, oft mehr verheißen. Es gilt darum, hier ihre reale Leistungsfähigkeit darzutun und in den Gesamtzusammenhang von Wirk- 
lichkeitsgestaltung durch PR zu stellen. Im Einzelnen ist da Folgendes hervorzuheben:

- Impression Management bzw. Image Control ist in der modernen Gesellschaft zentral geworden, weil deren Wirklichkeit auch in dieser Hinsicht mehr und mehr eine doppelte geworden ist, nämlich eine reale und eine imaginierte. Natürlich leben Menschen immer diese Doppelexistenz, aber erst wieder in Informations-, Kommunikationsoder Mediengesellschaften nach der religiösen Verdoppelung des mittelalterlichen irdischen Lebens konstituieren Images eine eigentliche Parallelwelt zu deren Originalen. Besonders markant prägt sich diese Verdoppelung im Wirtschaftssystem durch die Parallelisierung von Waren bzw. Gütern und Markenkultur aus. Nicht mehr die Waren selber werden beworben, sondern deren wiederum vom Kommunikationsstrategiesystem Werbung kreierten Images. Die Nähe von Werbung und PR und auch ihr analog beträchtlicher gesellschaftlicher Einfluss zeigen sich in beider Gestaltungsmacht einer wachsenden symbolischen Parallelwelt. Mittlerweile hat sich Image Control als generelles Branding, gesellschaftliche Positionszuweisung durch Markierung, auch in Politik und Kultur durchgesetzt. Gerade diese neue Allgegenwart von Images, die alle um Aufmerksamkeit und Akzeptanz konkurrieren, erschwert indes ihre öffentliche Durchsetzbarkeit und beschleunigt auch ihren Abbau.

- Dieser wird auch durch Techniken des Negative Campaigning befördert, allerdings nicht selten am falschen Objekt. Der alte Bumerangeffekt aus der Medienwirkungsforschung, dass also das Image des Urhebers von abwertenden, populär gesagt: Schmutzkampagnen selber Schaden leidet anstatt dasjenige des Zielobjekts, kann sich nämlich einstellen, wenn negative Kampagnen Normen der jeweiligen Streitkultur oder politische Fairnessregeln verletzen. In früheren $\mathrm{Ge}$ sellschaften scheint man, wie der Presserückschlag politischer Auseinandersetzungen verrät, klaglos mehr ausgeteilt und eingesteckt zu haben als im Zeitalter schrumpfender Frustrationstoleranz.

- Issues Management vollends, zumal im Zusammenhang mit Krisen und Konflikten, ist primär eine Aufgabe sachgerechter Problemidentifikation und -definition. Optimale PR antizipieren Krisen, z.B. des öffentlichen Vertrauens in ihre Klienten oder Anliegen, und handeln nicht erst, wenn dieses erschüttert ist. Dies setzt allerdings entsprechende Auftragsbereitschaft von Klienten voraus und bei den PRProfessionals umfassende Kenntnis von deren Problemen und auch Schwachstellen und überdies Einsicht in die Mechanismen gesellschaftlichen Wandels, antizipatorische Sensibilität dank entsprechender Beobachtungsinstrumente. So kann das Reputationskapital von 
Klienten zumindest einigermaßen bewahrt werden. $\mathrm{Zu}$ berïcksichtigen ist überdies stets, dass in Krisensituationen normale Symbiosen von Medien- und PR-Schaffenden von den ersteren meist aufgekündigt werden. Kritische Eigenaktivität demonstrierend, unterlaufen sie dann mit ihren Recherchen gerade die abwiegelnden PR-Strategien. $\mathrm{Zu}$ alledem müssen PR immer dem Umstand Rechnung tragen, dass Experten und Laien Krisen unterschiedlich definieren und insbesondere auch je andere Ursachen dafür geltend machen. Dies trifft erst recht auf die Wahrnehmung von Konflikten $z u$, in denen das Verhalten von Positions- und Gesinnungsgleichen notorisch positiver beurteilt wird als dasjenige der Gegenseite. Selbst Gewalt als besonders nachrichtenträchtiges Element von Konfliktaustragung wird ambivalent qualifiziert. Die wünschenswerten Wirklichkeiten, die Issues $\mathrm{Ma}$ nagement etablieren soll, sind mithin selber so umstritten wie Issues Management als PR-Strategie mit Risiken belastet ist.

Und das Fazit aus dieser Analyse? Was kann aus ihr gegebenenfalls für eine effiziente und zugleich verantwortungsbewusste PR-Kultur abgeleitet werden? Entsprechende Fragen werden ohnehin auch im PR-wissenschaftlichen und professionellen Schrifttum häufig abgehandelt, allerdings nicht konsequent im Hinblick auf die Gestaltung von Realität durch PR. Dabei ist deren Qualität für die Reputation und damit auch für das gegenwärtige und künftige Wirkungsvermögen professioneller Öffentlichkeitsarbeit entscheidend. Eine repräsentative Umfrage unter der deutschen Bevölkerung zum Image der PR-Branche ergab ja im Jahre 2003 nicht nur allgemein geringes Vertrauen in deren Repräsentanten, sondern im Speziellen, zumal von Seiten der Journalisten, Zweifel an der Verlässlichkeit ihrer Wirklichkeitsdefinitionen (Bentele 2005, S. 562). Abschließend soll daher versucht werden, auch deren normative Implikationen in eine These zu fassen:

\section{Definitionsverantwortung von PR}

PR-Schaffende müssen bei ihrem kommunikativen Handeln unterschiedlichen und auch widersprüchlichen Realitätsdefinitionen gerecht werden: denjenigen ihrer Auftraggeber, denjenigen der anvisierten Teilöffentlichkeiten und der von ihrer Berufsethik formulierten Verpflichtung zu Wahrhaftigkeit. Von der Qualität ihrer Auseinandersetzung mit Norm und Gegennorm hängt maßgeblich die Glaubwürdigkeit und damit auch der Erfolg ihrer Tätigkeit ab. Nur bei sorgfältiger Prüfung der Verträglichkeit der jeweils implizierten Normen operieren PR verantwortungsvoll. 
Die Berufskultur der Public Relations hat in diesem Sinne, ständig konfrontiert mit Problemen von Normenverträglichkeit der PR-Arbeit (Saxer 1994), angeleitet auch von entsprechenden Ethikcodices, akzeptanzsteigernde Regeln des Wirklichkeitsbezugs entwickelt (Bentele 1994, S. 255f.):

- "PR-Kommunikation [soll] eher sachlich als positiv wertend sein." - „Für die Organisation negative Informationen sollen nicht verschwiegen werden."

- „Die aktive und symbolische Darstellung von Images muss ihre Entsprechung in der Wahrnehmung des Unternehmens bei den Unternehmensangehörigen haben. Diskrepanz zwischen der Außenwahrnehmung (Außenimage) und der Innenwahrnehmung von Organisationen (internes Image) haben Unglaubwürdigkeitseffekte zur Folge. Interne human relations und externe PR müssen zu integraler Unternehmenskommunikation zusammenschließen."

Diese Adäquatheitsregeln verbürgen zumindest einigermaßen verlässliche Orientierungshilfen für verschiedene Akteurkategorien. Diese und weitere Regeln verantwortlicher PR-Kultur müssen allerdings vielfach gegen erhebliche Widerstände von PR-Auftraggebern, aber auch von Zielöffentlichkeiten durchgesetzt werden. Sollensvorstellungen durchwirken ja manifest oder latent auch schon das Wirklichkeitsverständnis von PR-Auftraggebern und Zielöffentlichkeiten und müssen auf ihre gegenseitige Verträglichkeit und auch auf diejenige mit den Normen der PR-Berufskultur geprüft werden. Eine etwaige Harmonisierung differierender Normen stellt dabei hohe Anforderungen an die strategische Kompetenz von PR-Spezialisten. Wenn Auftraggeber auf ein Zuviel an Schönfärberei dringen, bleibt für sie allerdings nur die Ablehnung des Mandats. Dafür können sie damit rechnen, dass ihre Klienten, Politiker oder Unternehmer, längerfristig durch das von ihnen für sie erwirkte vorteilhafte Image genötigt werden, dieses durch ihr Verhalten zu bestätigen, um ihrerseits Glaubwürdigkeit $\mathrm{zu}$ bewahren. Auch darin wird einmal mehr deutlich, wie komplex die wechselseitigen Beziehungen im Arbeitsfeld Realitätsgestaltung durch PR sind.

\section{Literatur}

Anders, Günther (1956): Die Welt als Phantom und Matrize. Philosophische Betrachtungen zu Radio und Fernsehen. Schaffhausen.

Bentele, Günter (1994): Public Relations und Wirklichkeit. Beitrag zu einer Theorie der Öffentlichkeitsarbeit. In: Ders./Hesse, Kurt (Hg.): Publizistik in der Gesellschaft. Festschrift für Manfred Rühl. Konstanz, S. 237-267. 
Bentele, Günter (2005): Ethische Anforderungen an Öffentlichkeitsarbeit. In: Ders./Fröhlich, Romy/Szyska, Peter (Hg.): Handbuch der Public Relations. Wissenschaftliche Grundlagen und berufliches Handeln. Mit Lexikon. Wiesbaden, S. 562-574.

Bentele, Günter (2006): Wirklichkeit. In: Ders./Brosius, Hans-Bernd/Jarren, Otfried (Hg.): Lexikon Kommunikations- und Medienwissenschaft. Wiesbaden, S. $311 f$.

Brosda, Carsten (1999): Aufstand nach der „Krönungsmesse“. Der SPD-Parteitag 1998 in Leipzig: Zur Inszenierung journalistischer Inszenierungskritik. In: Schicha, Christian/Ontrup, Rüdiger (Hg.): Medieninszenierungen im Wandel. Interdisziplinäre Zugänge. Münster, Hamburg, London, S. 199-213.

Dorsch-Jungsberger, Petra E./Roegele, Otto B./Stolte, Wolfgang (1985): Konfliktpotentiale im Nachrichtenproduktionsprozeß: Der Papstbesuch in der Bundesrepublik Deutschland. In: Publizistik, 30. Jg., H. 2-3, S. 280-298.

Meckel, Miriam (2002): Gibt es eigentlich die Wirklichkeit noch? Einige Thesen zu den Fakten und Fiktionen medialer Entgrenzung. In: Baum, Achim/Schmidt, Siegfried J. (Hg.): Fakten und Fiktionen. Über den Umgang mit Medienwirklichkeiten. Konstanz, S. 31-35.

Merten, Klaus/Westerbarkey, Joachim (1994): Public Opinion und Public Relations. In: Merten, Klaus/Schmidt, Siegfried J./Weischenberg, Siegfried (Hg.): Die Wirklichkeit der Medien. Eine Einführung in die Kommunikationswissenschaft. Opladen, S. 188-211.

Merten, Klaus (2002): Erzeugung von Fakten durch Reflexivisierung von Fiktionen. Strukturen der Ausdifferenzierung des Kommunikationssystems. In: Baum, Achim/Schmidt, Siegfried J. (Hg.): Fakten und Fiktionen. Über den Umgang mit Medienwirklichkeiten. Konstanz, S. 36-47.

Merten, Klaus (2005a): Zur Ausdifferenzienung der Mediengesellschaft. Wirklichkeitsmanagement als Suche nach Wahrheit. In: Arnold, Klaus/Neuberger, Chris toph (Hg.): Alte Medien - neue Medien. Theorieperspektiven, Medienprofile, Einsatzfelder. Festschrift für Jan Tonnemacher. Wiesbaden, S. 21-39.

Merten, Klaus (2005b): Konstruktivistischer Ansatz. In: Bentele, Gïnter/Fröhlich, Romy/Szyska, Peter (Hg.): Handbuch der Public Relations. Wissenschaftliche Grundlagen und berufliches Handeln. Mit Lexikon. Wiesbaden, S. 136-146.

Saxer, Ulrich (1994): Norm und Gegennorm: Probleme von Normenverträglichkeit in der PR-Arbeit. In: Armbrecht, Wolfgang/Zabel, Urs (Hg.): Normative Aspekte der Public Relations. Grundlegende Fragen und Perspektiven. Eine Einführung. Opladen, S. 195-224. 